HD-THEP-09-8

\title{
Non-Gaussianity beyond slow roll in multi-field inflation
}

\author{
Christian T. Byrnes 1, Gianmassimo Tasinato 2 \\ Institut für Theoretische Physik, Philosophenweg 16 and 19, 69120 Heidelberg, Germany
}

\begin{abstract}
We study the non-Gaussianity generated during multiple-field inflation. We provide an exact expression for the bispectrum parameter $f_{N L}$ which is valid beyond the slow-roll regime, valid for certain classes of inflationary models. We then study a new, exact multi-field inflationary model considering a case where the bispectrum grows to observable values at the end of inflation. We show that in this case the trispectrum is also large and may even provide the dominant signal of non-Gaussianity.
\end{abstract}

\section{Introduction}

The inflationary paradigm predicts an almost scale-invariant spectrum of nearly Gaussian perturbations, in excellent agreement with observations [1. On the other hand, many different models of inflation fit present day data very well, and further information is needed in order to test and exclude most of them. NonGaussianity, a signal of the non-linearity of the perturbations, has emerged in recent years as an important discriminator between various inflationary models [2, 3].

There are various known ways to generate large non-Gaussianity within the inflationary paradigm. Models of inflation with non-canonical kinetic terms are characterized by an equilateral shape of nonGaussianity, generated on sub-Hubble scales [4. Non-local inflation can also lead to large non-Gaussianity with local shape [5]. These examples are non-generic, since inflaton perturbations are usually close to Gaussian at Hubble exit [6, 7]. On the other hand, a local shape of non-Gaussianity can be generated during the evolution of fluctuations on super-horizon scales 3 . Models have been proposed to produce a large non-Gaussianity with this shape after inflation, such as inflationary models with an inhomogeneous end [9, 10, modulated preheating or reheating [11, and the curvaton scenario [12]. However all of these methods require the existence of additional, very light scalar fields, which play no role during inflation but have a significant effect on generating the primordial curvature perturbation after inflation ends. Arguably, models which generate large, primordial non-Gaussianity during inflation are therefore more economical.

Multi-field models of inflation, in which the curvature perturbation evolves at superhorizon scales, are able, at least in principle, to generate large non-Gaussian fluctuations during inflation. Most of the previous analyses of primordial non-Gaussianity in the multi-field case focused on separable Ansätze for the scalar field potential, either sum separable (for two-fields see [13, for an arbitrary number of fields see [14, 15]) or product separable [16], and rely on the slow-roll approximation. Examples in which $f_{N L}$ can grow large during slow-roll inflation for these classes of potentials were given [17, as well as the general requirements that the initial conditions of the background inflationary trajectory must satisfy. Numerical analyses have also been made, going beyond the assumption of separability and/or slow roll [18, 19, but no example of a model giving rise to a large non-Gaussianity at the end of inflation has been found in those cases.

\footnotetext{
${ }^{1}$ E-mail: c.byrnes@thphys.uni-heidelberg.de

${ }^{2}$ E-mail: g.tasinato@thphys.uni-heidelberg.de

${ }^{3}$ Different possible shape dependences of the bispectrum (3-point function) are considered in [8.
} 
In this paper, we consider models of inflation driven by multiple scalar fields with canonical kinetic terms. We study the properties of non-Gaussianity, parameterised by the quantity $f_{N L}$ characterising the bispectrum, generated by the evolution of scalar perturbations on super Hubble scales. We develop a new method that provides, for certain classes of models, analytical expressions for $f_{N L}$ valid in regimes beyond a slow-roll approximation. Our method is based on the first-order Hamilton-Jacobi formalism developed by Salopek and Bond [20, which allows us to express inflationary observables in the multi-field case, without having to focus on a slow-roll regime (see 21] for a similar application of this formalism to the single-field case).

The main idea on which our arguments are based is to focus on inflationary multi-field trajectories in which the Hubble rate, and not the potential, is separable. This approach allows us to analytically study cases where the non-Gaussian parameter $f_{N L}$ becomes large, in regimes in which the slow-roll parameters are enhanced after Hubble exit, but before inflation ends. In particular, we provide analytical equations that express the non-linearity parameter $f_{\mathrm{NL}}$, in terms of quantities that generalise the slow-roll parameters during inflation. By means of the same method, we are also able to find new, exact multi-field homogeneous solutions, that satisfy our Ansatz, for which a large amount of non-Gaussianity can be generated before the end of inflation. This allows us to apply the general formulae we find on a concrete example, that shows how the $f_{N L}$ parameter, and also both non-linearity parameters which characterise the trispectrum, can grow parametrically large as a function of quantities that characterise the model. In some cases the trispectrum through $g_{N L}$ can give the dominant signal of non-Gaussianity.

The plan of this paper is as follows: first we briefly recap the $\delta N$ formalism, which allows us to compute the evolution of perturbations on large scales. In section 3 we present a method to calculate $f_{N L}$ exactly for some models, by using the Hamilton-Jacobi equations. In section 4 we consider some explicit multi-field models of inflation for which we find exact solutions, and in one case we show that the non-Gaussianity can become observably large by the end of inflation. Finally we conclude in section 5 .

\section{Brief recap of $\delta N$ approach to evolution of perturbations}

In this paper we use the so-called $\delta N$ approach to study the evolution of curvature perturbations. This formalism has been found to be convenient for studying non-Gaussianity produced during inflation, and has been implemented in several previous studies. In this section we give a very brief review of the formulae necessary for the following discussion.

The starting point is the expression for the number of e-foldings of expansion, given by

$$
N=\int_{t_{*}}^{t_{c}} H(t) d t
$$

evaluated from an initial spatially flat hypersurface to a final uniform density hypersurface. The perturbation in the number of $e$-foldings, $\delta N$, is the difference between the curvature perturbations on the initial and final hypersurfaces. We take the initial time to be Hubble exit during inflation, denoted by $t_{*}$. The final time $t_{c}$ corresponds to a uniform density hypersurface just before the end of inflation. The curvature perturbation is given by $[22,23]$

$$
\zeta=\delta N=\sum_{I} N_{, I} \delta \varphi_{I *}+\frac{1}{2} \sum_{I J} N_{, I J} \delta \varphi_{I *} \delta \varphi_{J *}+\cdots
$$

where $N_{I}=\partial N /\left(\partial \varphi_{*}^{I}\right)$ and the index $I$ runs over all of the fields. The power spectrum and bispectrum are 
defined (in Fourier space) by 4

$$
\begin{aligned}
\left\langle\zeta_{\mathbf{k}_{1}} \zeta_{\mathbf{k}_{\mathbf{2}}}\right\rangle & \equiv(2 \pi)^{3} \delta^{3}\left(\mathbf{k}_{\mathbf{1}}+\mathbf{k}_{\mathbf{2}}\right) \frac{2 \pi^{2}}{k_{1}^{3}} \mathcal{P}_{\zeta}\left(k_{1}\right), \\
\left\langle\zeta_{\mathbf{k}_{1}} \zeta_{\mathbf{k}_{2}} \zeta_{\mathbf{k}_{3}}\right\rangle & \equiv(2 \pi)^{3} \delta^{3}\left(\mathbf{k}_{\mathbf{1}}+\mathbf{k}_{\mathbf{2}}+\mathbf{k}_{\mathbf{3}}\right) B_{\zeta}\left(k_{1}, k_{2}, k_{3}\right) .
\end{aligned}
$$

From this, we can define three quantities of key observational interest, respectively the spectral index, the tensor-to-scalar ratio and the non-linearity parameter

$$
\begin{aligned}
n_{\zeta}-1 & \equiv \frac{\partial \log \mathcal{P}_{\zeta}}{\partial \log k} \\
r & =\frac{\mathcal{P}_{T}}{\mathcal{P}_{\zeta}}=\frac{8 \mathcal{P}_{*}}{M_{P}^{2} \mathcal{P}_{\zeta}} \\
f_{\mathrm{NL}} & =\frac{5}{6} \frac{k_{1}^{3} k_{2}^{3} k_{3}^{3}}{k_{1}^{3}+k_{2}^{3}+k_{3}^{3}} \frac{B_{\zeta}\left(k_{1}, k_{2}, k_{3}\right)}{4 \pi^{4} \mathcal{P}_{\zeta}^{2}} .
\end{aligned}
$$

Here, $\mathcal{P}_{*}$ is the power spectrum of the scalar field fluctuations and $\mathcal{P}_{T}=8 \mathcal{P}_{*}=8 H_{*}^{2} /\left(4 \pi^{2} M_{P}^{2}\right)$ is the power spectrum of the tensor fluctuations. Both the spectra are calculated at the end of inflation and we will ignore any evolution after this time. As defined above, $f_{\mathrm{NL}}$ is shape dependent, but it has been shown that the shape dependent part is much less than unity [6.7]. The ideal CMB experiment is only expected to reach a precision of $f_{\mathrm{NL}}$ around unity [24], so we will calculate the shape independent part of $f_{\mathrm{NL}}$, denoted by $f_{\mathrm{NL}}^{(4)}$ in [13, 16]. Whenever the non-Gaussianity is large, $\left|f_{\mathrm{NL}}\right| \gtrsim 1$ as in the examples we are going to consider, we can associate $f_{\mathrm{NL}}^{(4)} \simeq f_{\mathrm{NL}}$. This ( $k$ independent) part of $f_{\mathrm{NL}}$ and the spectral index can be calculated by the $\delta N$ formalism,

$$
\begin{aligned}
\mathcal{P}_{\zeta} & =\sum_{I} N_{, I}^{2} \mathcal{P}_{*}, \\
n_{\zeta}-1 & =-2 \epsilon^{*}+\frac{2}{H} \frac{\sum_{I J} \dot{\varphi}_{J} N_{, J I} N_{, I}}{\sum_{K} N_{, K}^{2}}, \\
f_{\mathrm{NL}}^{(4)} & =\frac{5}{6} \frac{\sum_{I J} N_{, I J} N_{, I} N_{, J}}{\left(\sum_{I} N_{, I}^{2}\right)^{2}} .
\end{aligned}
$$

where $\epsilon_{\star} \equiv-\dot{H}_{\star} / H_{\star}^{2}$, and $H_{\star}=H\left(t=t_{\star}\right)$. In the next sections, we will find expressions for the previous quantities, valid also beyond a slow-roll approximation, for certain classes of multi-field inflationary models.

\section{The method}

In this section we present a method which allows us to obtain simple and compact expressions for the spectral index $n_{\zeta}$ and the parameter $f_{\mathrm{NL}}^{(4)}$, in terms of quantities associated with the homogeneous solution under consideration. The main advantage of this method is that we do not rely on any approximation in order to find our results. The final formulae then allow one to analytically study interesting regimes in field space, that cannot be probed within the slow-roll approximation, usually implemented when studying the evolution of perturbations during inflation.

We start in section 3.1 by reformulating the equations of motion as first order Hamilton-Jacobi equations, following [20]. By implementing a suitable Ansatz, this formulation allows one to define two quantities that play an important role for studying the evolution of fluctuations, that we start discussing from section 3.2 .

\footnotetext{
${ }^{4}$ Note that different sign conventions of $f_{N L}$ have been used. We use the same sign convention as WMAP [1], which is opposite to that used in 13, 16.
} 


\subsection{The homogeneous equations}

We consider a system of two scalar fields $\phi$ and $\chi$ with canonical kinetic terms, minimally coupled to gravity. The action, setting $M_{P l}^{2}=8 \pi G=1$, where $G$ is Newton's gravitational constant, is

$$
S=\int d^{4} x \sqrt{-g}\left[\frac{R}{2}-\frac{1}{2} g^{\mu \nu} \partial_{\mu} \phi \partial_{\nu} \phi-\frac{1}{2} g^{\mu \nu} \partial_{\mu} \chi \partial_{\nu} \chi-W(\phi, \chi)\right] .
$$

We focus on homogeneous, classical trajectories, adopting a FRW Ansatz for the metric and flat three dimensional spatial slices:

$$
d s^{2}=-d t^{2}+a(t)^{2}\left(d x_{1}^{2}+d x_{2}^{2}+d x_{3}^{2}\right) .
$$

Defining the Hubble parameter as $H \equiv \frac{\dot{a}}{a}$, the equations of motion are

$$
\begin{aligned}
H^{2} & =\frac{1}{3} W(\phi, \chi)+\frac{2}{3}\left[\left(\frac{\partial H}{\partial \phi}\right)^{2}+\left(\frac{\partial H}{\partial \chi}\right)^{2}\right], \\
\dot{\phi} & =-2 \frac{\partial H}{\partial \phi} \quad, \quad \dot{\chi}=-2 \frac{\partial H}{\partial \chi} .
\end{aligned}
$$

We concentrate on solutions satisfying the following Ansatz for the Hubble parameter $H(\phi, \chi)$ :

$$
H(\phi, \chi)=H^{(1)}(\phi)+H^{(2)}(\chi)
$$

that is, we demand that it can be split as a sum 5 of two pieces, each one depending on a single field 6 . Notice that this is different with respect to the requirement of separable potentials usually done in the literature [13]. Indeed, given that the Hubble parameter appears squared in the left hand side of eq. (13), it is easy to see that the potential cannot be written as a sum of pieces, each depending on a single field.

This separability Ansatz allows to define two quantities that characterise each homogeneous trajectory that satisfies eqs (13)-(14). The first is the number of e-foldings at time $t_{c}$, that can be expressed as

$$
N\left(t_{c}, t_{\star}\right)=\int_{t_{\star}}^{t_{c}} H(t) d t=-\frac{1}{2} \int_{\phi_{\star}}^{\phi_{c}} \frac{H^{(1)}}{H_{, \phi}^{(1)}} d \phi-\frac{1}{2} \int_{\chi_{\star}}^{\chi_{c}} \frac{H^{(2)}}{H_{, \chi}^{(2)}} d \chi
$$

where we define $H_{, \phi} \equiv \frac{\partial H(\phi)}{\partial \phi}$ and analogously for $\chi$. The second is a quantity $\mathcal{C}$, conserved along each homogenous trajectory,

$$
\mathcal{C}=-\int \frac{d \phi}{H_{, \phi}^{(1)}}+\int \frac{d \chi}{H_{, \chi}^{(2)}}
$$

whose definition resembles an analogous quantity used in [13].

While the number of e-foldings $N$ parameterises the evolution along a given homogeneous trajectory, $\mathcal{C}$ characterises the motion orthogonal to it. We will see these quantities are useful in order to analyse the evolution of fluctuations at superhorizon scales, in a way similar to what is done in [13. The main advantage

\footnotetext{
${ }^{5}$ Hence we follow an analysis related to [13. In an analogous way, one could also consider a situation in which the Hubble parameter splits as a product of single-field pieces [16.

${ }^{6}$ In principle, the Hubble parameter $H$ is a function $H\left(\varphi^{(k)}(t), t\right)$, with $\varphi^{(1)}=\phi$ and $\varphi^{(2)}=\chi$. However, by means of eq. (13) and (14), we have

$$
\left(\frac{\partial H^{2}}{\partial t}\right)_{\mid \varphi^{(k)}=\text { const }}=\frac{4}{3} \frac{\partial H}{\partial \varphi^{(k)}}\left[\frac{\partial^{2} H}{\partial \varphi^{(k)} \partial t}\right]_{\mid \varphi^{(k)}=\text { const }}=-\frac{2}{3} \frac{\partial H}{\partial \varphi^{(k)}}\left[\frac{\partial^{2} \varphi^{(k)}}{\partial t^{2}}\right]_{\mid \varphi^{(k)}=\text { const }}=0
$$
}

So that we have always $H=H\left(\varphi^{(k)}(t)\right)$ without additional dependence on time [20]. 
of our Ansatz (15) for the Hubble parameter is that the evolution of perturbations can be studied without having to rely on any approximation such as slow roll. The formulae that we are going to determine, then, can be applied also in regimes where slow-roll conditions are violated: this fact will be of crucial importance for the applications that we discuss in the second part of the paper.

\subsection{Evolution of perturbations}

As explained in section 2, we intend to follow the evolution of the curvature perturbation between an initially flat slice at time $t_{\star}$ (corresponding to Hubble exit) up to a final uniform density hypersurface at time $t_{c}$. To calculate the scalar spectral index and the $f_{\mathrm{NL}}$ parameter, as in eqs. (9) and (10), we have to compute the first and second derivatives of the number of e-foldings along the initial values for the fields $\phi_{\star}$ and $\chi_{\star}$. In order to do this, we use the properties of the functions $N\left(t_{\star}, t_{c}\right)$ and $C\left(\phi_{\star}, \chi_{\star}\right)$, and we closely follow the arguments developed in [13] transposing them to our case of separable Hubble parameter. We can write

$$
\begin{aligned}
d N & =\frac{1}{2}\left[\frac{H_{\star}^{(1)}}{H_{, \phi_{\star}}^{(1)}}-\frac{H_{c}^{(1)}}{H_{, \phi_{c}}^{(1)}} \frac{\partial \phi_{c}}{\partial \phi_{\star}}-\frac{H_{c}^{(2)}}{H_{, \chi_{c}}^{(2)}} \frac{\partial \chi_{c}}{\partial \phi_{\star}}\right] d \phi_{\star} \\
& +\frac{1}{2}\left[\frac{H_{\star}^{(2)}}{H_{, \chi_{\star}}^{(2)}}-\frac{H_{c}^{(2)}}{H_{, \chi_{c}}^{(2)}} \frac{\partial \chi_{c}}{\partial \chi_{\star}}-\frac{H_{c}^{(1)}}{H_{, \phi_{c}}^{(1)}} \frac{\partial \phi_{c}}{\partial \chi_{\star}}\right] d \chi_{\star} .
\end{aligned}
$$

We then need the derivatives of $\phi_{c}, \chi_{c}$ along the initial values of the fields. The following relations hold

$$
\begin{aligned}
& d \phi_{c}=\frac{d \phi_{c}}{d \mathcal{C}}\left(\frac{\partial \mathcal{C}}{\partial \phi_{\star}} d \phi_{\star}+\frac{\partial \mathcal{C}}{\partial \chi_{\star}} d \chi_{\star}\right), \\
& d \chi_{c}=\frac{d \chi_{c}}{d \mathcal{C}}\left(\frac{\partial \mathcal{C}}{\partial \phi_{\star}} d \phi_{\star}+\frac{\partial \mathcal{C}}{\partial \chi_{\star}} d \chi_{\star}\right),
\end{aligned}
$$

since $\phi_{c}$ and $\chi_{c}$ are functions of the conserved quantity $\mathcal{C}$. Also, we have

$$
\frac{\partial \mathcal{C}}{\partial \phi_{\star}}=-\frac{1}{H_{, \phi_{\star}}^{(1)}} \quad, \quad \frac{\partial \mathcal{C}}{\partial \chi_{\star}}=\frac{1}{H_{, \chi_{\star}}^{(2)}} .
$$

By definition, the time $t=t_{c}$ corresponds to a hypersurface of constant energy density, so that $H\left(\phi_{c}, \chi_{c}\right)=$ const in a flat universe. This implies

$$
\frac{d \phi_{c}}{d \mathcal{C}} H_{, \phi_{c}}^{(1)}+\frac{d \chi_{c}}{d \mathcal{C}} H_{, \chi_{c}}^{(2)}=0
$$

On the other hand, differentiating condition (17) along $\mathcal{C}$ one gets

$$
1=-\frac{d \phi_{c}}{d \mathcal{C}} \frac{1}{H_{, \phi_{c}}^{(1)}}+\frac{d \chi_{c}}{d \mathcal{C}} \frac{1}{H_{, \chi_{c}}^{(2)}} .
$$

By plugging (23) inside (22) one can extract

$$
\frac{d \phi_{c}}{d \mathcal{C}}=-\frac{H_{, \phi_{c}}^{(1)}\left(H_{, \chi_{c}}^{(2)}\right)^{2}}{\left(H_{, \phi_{c}}^{(1)}\right)^{2}+\left(H_{, \chi_{c}}^{(2)}\right)^{2}} .
$$


This, together with (17), is enough to get

$$
\frac{\partial \phi_{c}}{\partial \phi_{\star}}=\frac{d \phi_{c}}{d \mathcal{C}} \frac{\partial C}{\partial \phi_{\star}}=\frac{1}{H_{, \phi_{c}}^{(1)} H_{, \phi_{\star}}^{(1)}}\left(\frac{1}{\left(H_{, \phi_{c}}^{(1)}\right)^{2}}+\frac{1}{\left(H_{, \chi_{c}}^{(2)}\right)^{2}}\right)^{-1}
$$

and analogous formulae for the other derivatives of the fields. At this point it is convenient to define the following quantities:

$$
\begin{aligned}
\delta^{\phi}=\left(\frac{H_{, \phi}^{(1)}}{H}\right)^{2}, & \delta^{\chi}=\left(\frac{H_{, \chi}^{(2)}}{H}\right)^{2}, \\
\gamma^{\phi}=\frac{H_{, \phi \phi}^{(1)}}{H} \quad, & \gamma^{\chi}=\frac{H_{, \chi \chi}^{(2)}}{H},
\end{aligned}
$$

and $\delta=\delta^{\phi}+\delta^{\chi}$. Although their definition resembles the corresponding one for the usual slow roll parameters $\epsilon$ and $\eta$, they do not coincide with them when taking a slow roll limit 7 . We assume that the quantities $\delta$ and $\gamma$ are much smaller than unity at Hubble exit $t=t_{\star}$, in order to have a reliable expansion for $\delta N$ as explained in section (2). During inflation, by definition, we have to ensure that the quantity $\epsilon_{H} \equiv-\dot{H} / H^{2}$ is smaller than one. On the other hand,

$$
\epsilon_{H}=-\frac{1}{H^{2}}\left(H_{, \phi}^{(1)} \dot{\phi}+H_{, \chi}^{(2)} \dot{\chi}\right)=2 \delta,
$$

implying that $\delta$ must remain small during the inflationary period. The quantities $\gamma$ can however become large during inflation: this property will be very important in the following discussion.

By means of these quantities, we can write

$$
\begin{array}{lll}
\frac{\partial \phi_{c}}{\partial \phi_{\star}}=\frac{H_{c}}{H_{\star}} \frac{\delta_{c}^{\chi}}{\delta_{c}}\left(\frac{\delta_{c}^{\phi}}{\delta_{\star}^{\phi}}\right)^{\frac{1}{2}}, & \frac{\partial \phi_{c}}{\partial \chi_{\star}}=-\frac{H_{c}}{H_{\star}} \frac{\delta_{c}^{\chi}}{\delta_{c}}\left(\frac{\delta_{c}^{\phi}}{\delta_{\star}^{\chi}}\right)^{\frac{1}{2}}, \\
\frac{\partial \chi_{c}}{\partial \phi_{\star}}=-\frac{H_{c}}{H_{\star}} \frac{\delta_{c}^{\phi}}{\delta_{c}}\left(\frac{\delta_{c}^{\chi}}{\delta_{\star}^{\phi}}\right)^{\frac{1}{2}}, & \frac{\partial \chi_{c}}{\partial \chi_{\star}}=\frac{H_{c}}{H_{\star}} \frac{\delta_{c}^{\phi}}{\delta_{c}}\left(\frac{\delta_{c}^{\chi}}{\delta_{\star}^{\chi}}\right)^{\frac{1}{2}} .
\end{array}
$$

We now have all the instruments to compute the derivatives of the number of e-foldings. The first derivatives result

$$
\frac{\partial N}{\partial \phi_{\star}}=\frac{1}{2 \sqrt{\delta_{\star}^{\phi}}} \frac{H_{\star}^{(1)}+Z_{c}}{H_{\star}}, \quad \frac{\partial N}{\partial \chi_{\star}}=\frac{1}{2 \sqrt{\delta_{\star}^{\chi}}} \frac{H_{\star}^{(2)}-Z_{c}}{H_{\star}},
$$

where

$$
Z_{c}=\frac{H_{c}^{(2)} \delta_{c}^{\phi}-H_{c}^{(1)} \delta_{c}^{\chi}}{\delta_{c}}
$$

\footnotetext{
${ }^{7}$ It is nevertheless simple to work out the relation with the slow roll parameters, defined as

$$
\epsilon^{\phi} \equiv \frac{1}{2}\left(\frac{W_{, \phi}}{W}\right)^{2} \quad \eta^{\phi \phi}=\frac{W_{, \phi \phi}}{W}
$$
}

and analogously for $\chi$. In a slow-roll regime, one finds

$$
\delta^{\phi} \simeq \frac{\epsilon^{\phi}}{2} \quad \gamma^{\phi} \simeq \frac{\eta^{\phi \phi}-\epsilon^{\phi}}{2} .
$$


This quantity $Z_{c}$ controls the evolution of the curvature perturbation at superhorizon scales, as expected in multi-field inflationary models.

The calculation of the second derivatives yields

$$
\begin{aligned}
\frac{\partial^{2} N}{\partial \phi_{\star}^{2}} & =\frac{1}{2}-\frac{\gamma_{\star}^{\phi}}{2 \delta_{\star}^{\phi}} \frac{H_{\star}^{(1)}+Z_{c}}{H_{\star}}+\frac{1}{2 H_{\star} \sqrt{\delta_{\star}^{\phi}}} \frac{\partial Z_{c}}{\partial \phi_{\star}}, \\
\frac{\partial^{2} N}{\partial \chi_{\star}^{2}} & =\frac{1}{2}-\frac{\gamma_{\star}^{\chi}}{2 \delta_{\star}^{\chi}} \frac{H_{\star}^{(2)}-Z_{c}}{H_{\star}}-\frac{1}{2 H_{\star} \sqrt{\delta_{\star}^{\chi}}} \frac{\partial Z_{c}}{\partial \chi_{\star}}, \\
\frac{\partial^{2} N}{\partial \chi_{\star} \partial \phi_{\star}} & =\frac{1}{2 H_{\star} \sqrt{\delta_{\star}^{\phi}}} \frac{\partial Z_{c}}{\partial \chi_{\star}}=-\frac{1}{2 H_{\star} \sqrt{\delta_{\star}^{\chi}}} \frac{\partial Z_{c}}{\partial \phi_{\star}} .
\end{aligned}
$$

We now have all the necessary quantities for evaluating the expressions discussed in section 2 ,

\subsection{Curvature perturbation and non-Gaussianities during inflation}

We can use eqs. (9) and (10) to calculate the scalar spectral index during inflation, as well as the non-linear parameter $f_{\mathrm{NL}}$. In order to do this, it is convenient to introduce two new variables

$$
u \equiv \frac{H_{\star}^{(1)}+Z_{c}}{H_{\star}} \quad, \quad v \equiv \frac{H_{\star}^{(2)}-Z_{c}}{H_{\star}},
$$

with the property that $u+v=1$. By means of these and of the results of the previous section, the spectral index for the curvature can be straightforwardly calculated from eq. (91), giving

$$
n_{\zeta}-1=-4 \delta_{\star}-4 \frac{u\left(1-\frac{\gamma_{\star}^{\phi}}{\delta_{\star}^{\phi}} u\right)+v\left(1-\frac{\gamma_{\star}^{\chi}}{\delta_{\star}^{\chi}} v\right)}{\frac{u^{2}}{\delta_{\star}^{\phi}}+\frac{v^{2}}{\delta_{\star}^{\chi}}} .
$$

Notice that, since it depends on the parameters $u$ and $v$, it changes with time also beyond horizon exit, due to the correlation between entropy and adiabatic perturbations. On the other hand, if $\delta_{\star}$ and $\gamma_{\star}$ are small, the spectral index normally remains close to one during the entire inflationary period.

We pass then to calculate $f_{\mathrm{NL}}^{(4)}$, the parameter on which we are more interested in. We start by introducing the quantity

$$
\mathcal{A}=-\frac{H_{c}^{2}}{H_{\star}^{2}} \frac{\delta_{c}^{\phi} \delta_{c}^{\chi}}{\delta_{c}}\left(\frac{1}{2}-\frac{\gamma_{c}^{s s}}{\delta_{c}}\right)
$$

where we define

$$
\gamma^{s s}=\left(\delta^{\chi} \gamma^{\phi}+\delta^{\phi} \gamma^{\chi}\right) / \delta
$$

One can then express the value for the $f_{N L}^{(4)}$ by plugging the previously obtained results in formula (10), finding 8

$$
\frac{6}{5} f_{N L}^{(4)}=2 \frac{\frac{u^{2}}{\delta_{\star}^{\phi}}\left(1-\frac{\gamma_{\star}^{\phi}}{\delta_{\star}^{\phi}} u\right)+\frac{v^{2}}{\delta_{\star}^{\chi}}\left(1-\frac{\gamma_{\star}^{\chi}}{\delta_{\star}^{\chi}} v\right)+2\left(\frac{u}{\delta_{\star}^{\phi}}-\frac{v}{\delta_{\star}^{\chi}}\right)^{2} \mathcal{A}}{\left(\frac{u^{2}}{\delta_{\star}^{\phi}}+\frac{v^{2}}{\delta_{\star}^{\chi}}\right)^{2}}
$$

\footnotetext{
${ }^{8}$ Notice the formal similarity with equation $(68)$ in 13 . The meaning of the various quantities, however, is different with respect to that paper. Various numerical factors are also different.
} 
This is one of the main results of our paper. We reiterate that this formula is exact and is not based on a slow-roll expansion. Notice that the first two terms of eq. (38) are suppressed by $\delta_{\star}^{\phi}$ or $\delta_{\star}^{\chi}$, and these quantities are less than unity since they are calculated at horizon exit. Only the last term proportional to $\mathcal{A}$ is not suppressed by quantities evaluated at horizon crossing. It depends on the quantity $\gamma_{c}^{s s}$, that can become much bigger than one during inflation, rendering the size of $\mathcal{A}$ large enough to enhance $f_{N L}$ to observable values. Our formalism allows to study this regime in a reliable, analytical fashion, contrary to an analysis relying on a slow-roll approximation. It is also possible to have a large $f_{N L}$ while all slow-roll parameters are small, if the ratio of some of the parameters is very large and the background trajectory curves in an appropriate way [17] (see also [25,26]). We note in agreement with [13] that if one of the fields has reached a minimum so $\dot{\phi}=0$ or $\dot{\chi}=0$ at the end of inflation then $\mathcal{A}=0$ and the non-Gaussianity at the end of inflation will be small. Therefore any model of inflation with a separable potential or separable Hubble factor with a large non-Gaussianity present at the end of inflation must have both fields still evolving, and therefore the presence of isocurvature modes. It would therefore also be interesting to study the evolution of the perturbations after inflation, to see if this leads to an important change in the observables [27.

In the next section, we apply our general results to various examples of inflationary trajectories, showing in concrete examples that our formulae can analytically probe regions in field space where the non-linear parameter $f_{\mathrm{NL}}^{(4)}$ is enhanced to large values.

\section{Exact solutions}

Our system of first order equations, discussed in section 3.1. also allows one to find new exact inflationary trajectories in the multi-field case, provided that the scalar potential satisfies some conditions. There are very few examples of multiple field exact solutions in the literature 28. In this section, we present two classes of new exact solutions, with the aim to study the evolution of non-Gaussianities in each example. In the first example, section 4.1, we analyse a quadratic potential, showing that in this case the non-gaussian parameter $f_{\mathrm{NL}}^{(4)}$ always remains small during the inflationary regime. In the second example, section 4.2, we consider a combination of exponential potentials. In this case, non-Gaussianities can get enhanced to observable values, depending on the choice of the parameters in the potential.

\subsection{Quadratic potential: no enhancement of $f_{\mathrm{NL}}$}

We first present an example of exact configuration that does not lead to enhanced non-Gaussianities, since the quantity $\mathcal{A}$ defined in eq. (36) does not increase much during inflation.

Consider a quadratic potential

$$
W=A+B \phi+C \chi+D \phi^{2}+E \chi^{2}+F \phi \chi
$$

where the constants $B, C$ and $F$ are related to the other quantities by

$$
\begin{aligned}
B^{2} & =\frac{8 D}{3}\left(\frac{3}{2} A+D+E\right), \\
C^{2} & =\frac{8 E}{3}\left(\frac{3}{2} A+E+D\right), \\
F^{2} & =4 D E .
\end{aligned}
$$

The equations of motion can be solved choosing a Hubble parameter of the form

$$
H=H_{0}+H_{1} \phi+H_{2} \chi,
$$


with

$$
H_{0}^{2}=\frac{2}{9}\left(\frac{3}{2} A+E+D\right), \quad H_{1}^{2}=\frac{D}{3}, \quad H_{2}^{2}=\frac{E}{3} .
$$

Setting for simplicity, and without loosing generality, $t_{\star}=0$, the equations have the solution

$$
\begin{aligned}
\phi(t) & =\phi_{\star}-2 H_{1} t, \quad \chi(t)=\chi_{\star}-2 H_{2} t, \\
a(t) & =a_{0} e^{\left(H_{0}+H_{1} \phi_{\star}+H_{2} \chi_{\star}\right) t-\left(H_{1}^{2}+H_{2}^{2}\right) t^{2}} .
\end{aligned}
$$

Notice that, although the solution is well defined for all $t$, at sufficiently large times the Hubble parameter becomes negative and the universe starts contracting. This point is, in any case, well beyond the end of the inflationary era, and we do not need to discuss it in our analysis. Inflation ends when the parameter $\epsilon_{H}$ given by

$$
\epsilon_{H}(t)=-\dot{H} / H^{2}=\frac{2\left(H_{1}^{2}+H_{2}^{2}\right)}{\left[H_{0}+H_{1} \phi_{\star}+H_{2} \chi_{\star}-2\left(H_{1}^{2}+H_{2}^{2}\right) t\right]^{2}}=2 \delta
$$

becomes unity. Notice that the equations of motion, and eq. (47), allows one to rewrite the expression for the potential in a suggestive form

$$
W=3 H^{2}-2\left(H_{1}^{2}+H_{2}^{2}\right)=3 H^{2}\left(1-\frac{\epsilon_{H}}{3}\right)
$$

ensuring that during inflation this quantity remains positive definite.

In order to rely on the expansion for the number of e-foldings discussed in section 2, we impose that the quantities $\delta_{*}^{\phi, \chi}$ are small. From eq. (47), we learn that this is ensured as long as $H_{1}, H_{2} \ll H_{0}$. Notice that the $\gamma$ parameters in this example vanish identically: this simplifies the expression for the spectral index, that reduces to

$$
n_{\zeta}=1-4 \delta_{\star}-4 \frac{\delta_{\star}^{\phi} \delta_{\star}^{\chi}}{u^{2} \delta_{\star}^{\chi}+v^{2} \delta_{\star}^{\phi}}
$$

so $n_{\zeta}-1$ is proportional to terms suppressed by small $\delta_{\star}$ 's parameters. Another consequence of having $\gamma^{\phi, \chi}=0$, is the fact that the quantity $\mathcal{A}$ cannot increase much during inflation. So, following the discussion of the previous sections, we do not expect to find any enhancement of non-Gaussianities. Indeed, it is straightforward to get the following expressions for the parameters $u$ and $v: u=\delta_{\star}^{\phi} / \delta_{\star}, v=\delta_{\star}^{\chi} / \delta_{\star}$. A simple calculation then provides

$$
\begin{aligned}
\frac{6}{5} f_{N L} & =\frac{2 \delta_{\star}^{\phi} \delta_{\star}^{\chi}}{\left(\delta_{\star}^{\chi} u^{2}+\delta_{\star}^{\phi} v^{2}\right)}-2 \frac{\left(\delta_{\star}^{\chi} u-\delta_{\star}^{\phi} v\right)^{2}}{\left(\delta_{\star}^{\chi} u^{2}+\delta_{\star}^{\phi} v^{2}\right)^{2}} \frac{\delta_{\star}^{\phi} \delta_{\star}^{\chi}}{\delta_{\star}} \\
& =2 \delta_{\star},
\end{aligned}
$$

that then remains small and constant during inflation. This is not surprising since, from eq. (45), one learns that the inflationary trajectory follows a straight line in field space. To finish the discussion of this example, notice that spectral index and $f_{N L}$ are connected by the relation $n_{\zeta}-1=-\frac{24}{5} f_{N L}$.

\subsection{Exponential potential: enhancement of $f_{\mathrm{NL}}$ towards the end of inflation}

We discuss here an exact inflationary trajectory, for a potential based on a combination of exponentials that is instead able to produce large values of $f_{\mathrm{NL}}$ at the end of inflation. Noticeably, a potential with a similar form may be motivated in string theory, in the context of Kähler moduli inflation [29]. We start by 
presenting the inflationary trajectory, and showing how it can enhance the non-linearity parameter $f_{\mathrm{NL}}$. We discuss the physical interpretation of our findings at the end of this section.

The potential we consider is

$$
W(\phi, \chi)=U_{0}\left(1-A_{1} e^{-\alpha \phi}+A_{2} e^{-2 \alpha \phi}-B_{1} e^{-\beta \chi}+B_{2} e^{-2 \beta \chi}+\frac{A_{1} B_{1}}{2} e^{-\alpha \phi-\beta \chi}\right),
$$

where the parameters $\alpha$ and $\beta$ satisfy

$$
\alpha^{2}=\frac{3}{2}-\frac{6 A_{2}}{A_{1}^{2}}, \quad \beta^{2}=\frac{3}{2}-\frac{6 B_{2}}{B_{1}^{2}} .
$$

Since one may freely redefine the initial field values, we will assume that $\alpha, \beta>0$ without loss of generality [40], and we also assume that $A_{1}, A_{2}>09$.

The equations of motion discussed in section 3.1 admit the following solution for $H$ :

$$
H=\sqrt{\frac{U_{0}}{3}}\left(1-\frac{A_{1}}{2} e^{-\alpha \phi}-\frac{B_{1}}{2} e^{-\beta \chi}\right) .
$$

The overall factor to the potential $U_{0}$ can be freely chosen so that the amplitude of the scalar power spectrum matches the observed amplitude of perturbations in the CMB.

The solutions for the scalar fields read (from now on $H_{0}=\sqrt{U_{0} / 3}$ and we have again set $t_{\star}=0$ )

$$
\phi=\frac{1}{\alpha} \ln \left[e^{\alpha \phi_{\star}}-A_{1} \alpha^{2} H_{0} t\right], \quad \chi=\frac{1}{\beta} \ln \left[e^{\beta \chi_{\star}}-B_{1} \beta^{2} H_{0} t\right] .
$$

The scale factor results:

$$
a(t)=a_{0}\left(e^{\alpha \phi_{\star}}-\alpha^{2} A_{1} H_{0} t\right)^{\frac{1}{2 \alpha^{2}}}\left(e^{\beta \chi_{\star}}-\beta^{2} B_{1} H_{0} t\right)^{\frac{1}{2 \beta^{2}}} e^{H_{0} t} .
$$

Notice that the solution becomes singular at late times, when the scale factor vanishes and the field values diverge. The singularity, on the other hand, occurs well after inflation ends, and we will not need to discuss it in our analysis. The quantities $\delta$ and $\gamma$ read in this case

$$
\begin{aligned}
\delta^{\phi} & =\frac{H_{0}^{2}}{H^{2}} \frac{\alpha^{2} A_{1}^{2}}{4} e^{-2 \alpha \phi} & , & \delta^{\chi}=\frac{H_{0}^{2}}{H^{2}} \frac{\beta^{2} B_{1}^{2}}{4} e^{-2 \beta \chi}, \\
\gamma^{\phi} & =-\frac{H_{0}}{H} \frac{\alpha^{2} A_{1}}{2} e^{-\alpha \phi} & , & \gamma^{\chi}=-\frac{H_{0}}{H} \frac{\beta^{2} B_{1}}{2} e^{-\beta \chi} .
\end{aligned}
$$

Useful relations are

$$
\begin{aligned}
\gamma^{\phi} & =-\alpha \sqrt{\delta^{\phi}}, \quad \gamma^{\chi}=-\beta \sqrt{\delta \chi} \\
H & =\frac{H_{0}}{1-\frac{\gamma^{\phi}}{\alpha^{2}}-\frac{\gamma^{\chi}}{\beta^{2}}} .
\end{aligned}
$$

Notice that inflation occurs while

$$
\epsilon_{H}=2 \delta^{\phi}+2 \delta^{\chi}=2\left(\frac{\gamma^{\phi}}{\alpha}\right)^{2}+2\left(\frac{\gamma^{\chi}}{\beta}\right)^{2} \leq 1 .
$$

\footnotetext{
${ }^{9}$ We did not make these positivity assumptions in versions 1 and 2 of this paper, see the "Note added" at the end of the conclusions.
} 
In this example $\gamma^{\phi}$ and $\gamma^{\chi}$ can become much larger than unity at the end of inflation if $\alpha$ and/or $\beta$ are much greater than one. This corresponds to a break down in slow roll, although $\epsilon_{H}$ remains smaller than unity during the inflationary era.

We can assume that at the initial time $t=t_{\star}$, the gamma parameters are smaller than $\alpha$ and $\beta$, so that $H_{\star} \simeq H_{0}$. Using the formalism developed in section 3 , we call

$$
H^{(1)}(\phi)=\frac{H_{0}}{2}-\frac{A_{1} H_{0}}{2} e^{-\alpha \phi}=\frac{H_{0}}{2}\left(1-\frac{2 \sqrt{\delta_{c}^{\phi}}}{\alpha} \frac{H_{c}}{H_{0}}\right) \quad, \quad H^{(2)}(\chi)=H(\phi, \chi)-H^{(1)}(\phi)
$$

The number of e-folding at time $t_{c}$ reads

$$
\begin{aligned}
N\left(\phi_{c}, \chi_{c}\right) & =\frac{1}{2 \alpha}\left(\phi-\phi_{\star}\right)+\frac{1}{2 \beta}\left(\chi-\chi_{\star}\right)-\frac{1}{2 A_{1} \alpha^{2}}\left(e^{\alpha \phi}-e^{\alpha \phi_{\star}}\right)-\frac{1}{2 B_{1} \beta^{2}}\left(e^{\beta \chi}-e^{\beta \chi_{\star}}\right) \\
& =\ln \left(\frac{\delta_{\star}^{\phi}}{\delta_{c}^{\phi}} \frac{H_{\star}^{2}}{H_{c}^{2}}\right)^{\frac{1}{4 \alpha^{2}}}+\ln \left(\frac{\delta_{\star}^{\chi}}{\delta_{c}^{\chi}} \frac{H_{\star}^{2}}{H_{c}^{2}}\right)^{\frac{1}{4 \beta^{2}}}+\frac{1}{4}\left(\frac{1}{\left|\gamma_{\star}^{\phi}\right|}+\frac{1}{\left|\gamma_{\star}^{\chi}\right|}\right)-\frac{H_{0}}{4 H_{c}}\left(\frac{1}{\alpha \sqrt{\delta_{c}^{\phi}}}+\frac{1}{\beta \sqrt{\delta_{c}^{\chi}}}\right) .
\end{aligned}
$$

Focussing on the regime of large $\alpha$ and $\beta$, in which the first two terms in the previous expression are suppressed, the main contribution to the number of e-foldings comes from the terms that go like $1 / \gamma_{\star}$, as long as $\delta_{c}^{\phi}$ or $\delta_{c}^{\chi}$ are not too small (more on this later).

We now proceed to calculate the quantities necessary for estimating the non-linear parameter $f_{\mathrm{NL}}$. One finds that

$$
u=\frac{1}{2}+\frac{\delta^{\phi}-\delta^{\chi}}{2 \delta}-\frac{\sqrt{\delta^{\phi}}}{\alpha}+\frac{\sqrt{\delta^{\phi} \delta^{\chi}}}{\delta}\left(\frac{\sqrt{\delta^{\chi}}}{\alpha}-\frac{\sqrt{\delta^{\phi}}}{\beta}\right)
$$

and

$$
\gamma^{s s}=-\frac{\sqrt{\delta^{\phi} \delta^{\chi}}}{\delta}\left[\alpha \sqrt{\delta \chi}+\beta \sqrt{\delta^{\phi}}\right]
$$

The expression for $\mathcal{A}$, evaluated at $t=t_{c}$ is given by

$$
\mathcal{A}=-\frac{1}{\left(1-\frac{\sqrt{\delta_{c}^{\phi}}}{\alpha}-\frac{\sqrt{\delta_{c}^{\chi}}}{\beta}\right)^{2}}\left(\frac{\delta_{c}^{\phi} \delta_{c}^{\chi}}{\delta_{c}^{2}}\right)^{\frac{3}{2}}\left(\alpha \sqrt{\delta_{c}^{\chi}}+\beta \sqrt{\delta_{c}^{\phi}}+\frac{\delta_{c}^{2}}{\sqrt{\delta_{c}^{\phi} \delta_{c}^{\chi}}}\right) .
$$

Although the quantities $\delta_{c}^{\phi}$ and $\delta_{c}^{\chi}$ are smaller than one during inflation, on the other hand the parameters $\alpha$ and $\beta$ can be large; this allows the quantity $\mathcal{A}$ to become large during inflation, enhancing the value of $f_{\mathrm{NL}}^{(4)}$. We now show this with an explicit example.

\section{A concrete example}

As a simple, concrete example, we choose a regime in which the parameters $\alpha$ and $\beta$ are both large, let us say larger than some arbitrary quantity $R \gg 1$ with no physical meaning by itself. We note from (52) that we are therefore required to have $A_{2}<0$ and $B_{2}<0$. In this case the potential does not have a minimum, but inflation still ends through $\epsilon_{H}$ growing larger than unity, and we can trust our results in this regime. The potential would need modifications which apply after inflation in order for reheating to take place and these modifications may provide a minimum for the potential. However a study of this issue is beyond the scope of this work. We parameterise the values of the quantities $\delta_{c}^{\phi}$ and $\delta_{c}^{\chi}$ at the end of inflation as 


$$
\delta_{c}^{\phi}=\frac{1}{2 m} \quad, \quad \delta_{c}^{\chi}=\frac{1}{2 n} \quad \text { such that } \quad \frac{1}{m}+\frac{1}{n}=1 .
$$

Suppose that the quantities $m$ and $n$ are not too big. Namely, they satisfy the inequality $m, n \ll R^{2}$, such that the expression for the number of e-foldings, eq. (61), is dominated by the terms proportional to the inverse of $\gamma_{\star}$. The same inequality also implies that $H_{0} \simeq H_{\star} \simeq H_{c}$. We choose $\left|\gamma_{\star}^{\phi}\right|=\frac{1}{40 p},\left|\gamma_{\star}^{\chi}\right|=\frac{1}{40 q}$, such that we can write

$$
N_{\text {tot }}=60 \simeq 10(p+q) .
$$

In order to have 60 e-foldings, we have to choose $p+q=6$. This fixes the initial values for the fields $\phi_{\star}$ and $\chi_{\star}$, to the values

$$
\begin{aligned}
e^{\alpha \phi_{\star}} & =20 p \alpha^{2} A_{1}, \\
e^{\beta \chi_{\star}} & =20 q \beta^{2} B_{1} .
\end{aligned}
$$

The conditions (65) imply

$$
\begin{aligned}
& H_{0} t_{c}=20 p-\sqrt{\frac{m}{2 \alpha^{2}}}, \\
& H_{0} t_{c}=20 q-\sqrt{\frac{n}{2 \beta^{2}}} .
\end{aligned}
$$

Since $m$ and $n$ are much smaller than $R^{2}$, we expect $p \simeq q$. We hence have the simple relations

$$
\delta_{\star}^{\phi} \simeq \frac{1}{4 N_{t o t}^{2} \alpha^{2}}, \quad \delta_{\star}^{\chi} \simeq \frac{1}{4 N_{t o t}^{2} \beta^{2}}, \quad \gamma_{\star}^{\phi} \simeq \gamma_{\star}^{\chi} \simeq-\frac{1}{2 N_{t o t}} .
$$

From (6), (8) and (29) for the tensor-to-scalar ratio, and from (35) for the spectral index, we find

$$
\begin{aligned}
r & \simeq \frac{8}{N_{t o t}^{2}} \frac{1}{\frac{\alpha^{2}}{m^{2}}+\frac{\beta^{2}}{n^{2}}} \ll 10^{-3}, \\
n_{\zeta}-1 & \simeq-\frac{2}{N_{t o t}} \simeq-0.04 .
\end{aligned}
$$

So we have a completely negligible level of gravitational waves and a red spectral index in agreement with present day observations [1].

It is interesting to notice that for this choice of initial conditions we can write

$$
\begin{aligned}
& \phi \simeq \frac{1}{\alpha} \log \left[N_{t o t}-N(t)+\sqrt{\frac{m}{2 \alpha^{2}}}\right]+\frac{1}{\alpha} \log \left[\alpha^{2} A_{1}\right], \\
& \chi \simeq \frac{1}{\beta} \log \left[N_{t o t}-N(t)+\sqrt{\frac{n}{2 \beta^{2}}}\right]+\frac{1}{\beta} \log \left[\beta^{2} B_{1}\right],
\end{aligned}
$$

where we used eq. (61). $N(t)$ indicates the number of e-folds at time $t$, and $N_{\text {tot }}$ corresponds to the total number of e-folds (that as we mentioned we take of order sixty). We know that the terms inside the square roots, in the argument of the logarithms appearing in the previous equations, are much smaller than one since $m, n \ll R^{2}$. This implies that in a regime in which $N_{t o t}-N(t) \gtrsim 1$ we can write

$$
\beta \chi(t)=\alpha \phi(t)-\log \frac{\alpha^{2} A_{1}}{\beta^{2} B_{1}} .
$$

Since $\chi$ is proportional to $\phi$, we have a straight trajectory in field space, implying that we cannot have conversion of entropy into adiabatic modes [30], and consequently the curvature perturbation remains almost 


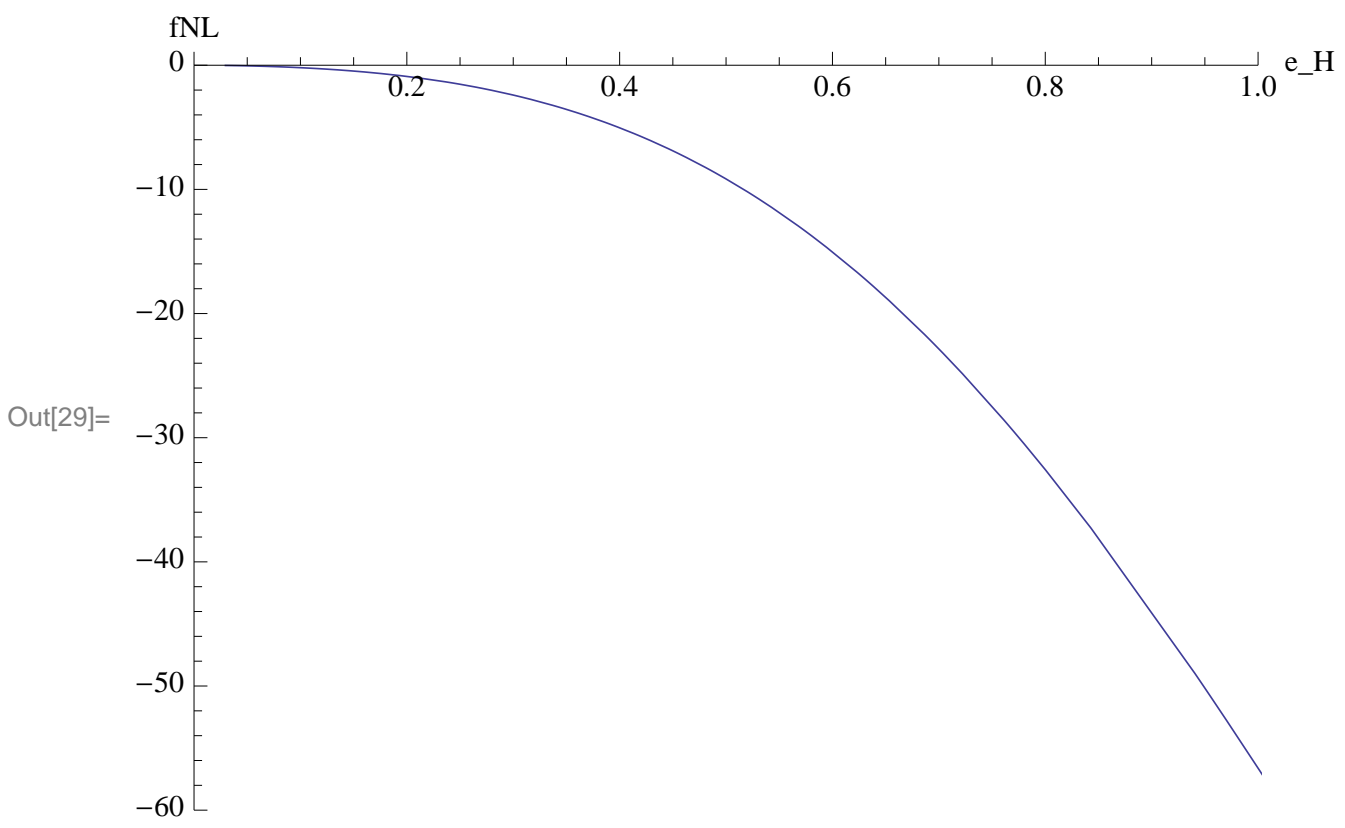

Figure 1: Plot showing $f_{N L}$ as given by (78) as a function of $\epsilon_{H}$ towards the end of inflation, for the values of the parameters given in the text. Inflation ends when $\epsilon_{H}=1$; for this example $f_{N L} \simeq-59$ at that time.

constant at superhorizon scales. In this regime, for the arguments we have seen in the previous sections, we cannot produce large non-Gaussianities in this context. However, towards the end of inflation, $N_{t o t}-N(t)<1$ and the trajectory acquires a sharp turn, since the terms inside the square roots in eqs. (74) and (75) start to give sizeable contributions. Precisely in this regime the curvature perturbation evolves at superhorizon scales, and parametrically large non-Gaussianities can be produced, as we are going to see now.

We can calculate $u$ and $v$ in this case, finding (from now on we neglect corrections of order $1 / R$ )

$$
u \simeq \frac{1}{2}+\frac{1}{2} \frac{n-m}{n+m}=2 \delta_{c}^{\phi}, \quad v \simeq \frac{1}{2}-\frac{1}{2} \frac{n-m}{n+m}=2 \delta_{c}^{\chi} .
$$

It is simple to check that the curvature spectral index $n_{\zeta}$ given in eq. (9) remains close to one during the entire inflationary period.

The parameter $\mathcal{A}$ becomes

$$
\mathcal{A} \simeq-\frac{1}{\sqrt{2}(m n)^{\frac{3}{2}}}\left(\frac{\alpha}{\sqrt{n}}+\frac{\beta}{\sqrt{m}}\right)
$$

The leading contribution to the quantity $f_{\mathrm{NL}}^{(4)}$ is

$$
\frac{6}{5} f_{\mathrm{NL}} \simeq 4\left(\frac{u \delta_{\star}^{\chi}-v \delta_{\star}^{\phi}}{u^{2} \delta_{\star}^{\chi}+v^{2} \delta_{\star}^{\phi}}\right)^{2} \mathcal{A} .
$$

When evaluated at the end of inflation, using (177) we find

$$
\frac{6}{5} f_{\mathrm{NL}} \simeq-2 \sqrt{2 m n}\left[\frac{\alpha^{2} n-\beta^{2} m}{\alpha^{2} n^{2}+\beta^{2} m^{2}}\right]^{2}\left(\frac{\alpha}{\sqrt{n}}+\frac{\beta}{\sqrt{m}}\right)
$$

We stress that this formula provide only the dominant contributions to $f_{N L}$. It is valid in the case $|\alpha|,|\beta|>$ $R \gg 1$, and $m, n<R^{2}$. 

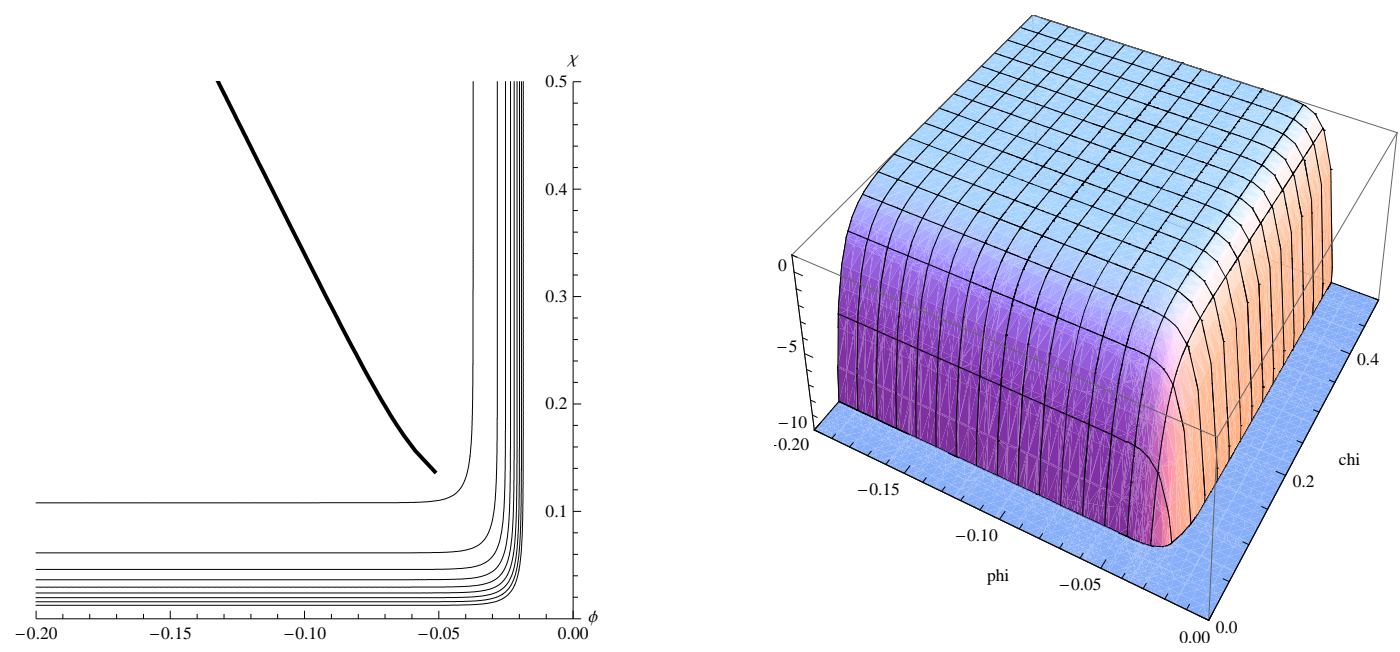

Figure 2: Left plot shows the trajectory considered for the parameters given after eq. (79) superimposed on a contour plot of the potential. The square on the trajectory indicates a point along the trajectory one $e$-folding before inflation ends as $\phi$ and $\chi$ roll towards zero. This shows that the fields roll much more quickly during the final stage of inflation, and the trajectory curves at the end as discussed in the text after (76). The right plot shows the potential for the same parameter values. Notice that inflation ends on the plateau long before the potential becomes negative.

As an explicit case, we take $\alpha=100, \beta=20, m=6, n=6 / 5$ and the initial conditions to satisfy (74) and (75) with $N_{t o t}=60$. Then, independently of the values of $A_{1}$ and $B_{1}$, we find $f_{N L} \simeq-58$ from the simplified formula (79), and $f_{N L} \simeq-53$ from the full formula (38). See figures 1 and 2. Notice that the results for $f_{N L}$ differ from each other by quantities of order $1 / R=1 / \beta$ as expected by the approximations we have made. At the end of inflation, the parameters are $\gamma^{\phi}=29, \gamma^{\chi}=-13$ and this shows that the slow-roll approximation has broken down, see footnote 7 .

Let us end with some considerations regarding our findings in this concrete example. Our expression for $f_{\mathrm{NL}}$ in eq. (79) depends on the initial values through $p$ and $q$, and this dependence drops out at leading order in $\alpha$. Hence $f_{N L}$ is independent of the number of $e$-foldings and scale independent 10 . This is easy to understand, since we have seen that, in our example, non-Gaussianity can be produced only towards the very end of inflation, when the trajectory has a sharp turn in field space.

Notice that our requirements of final values for the quantities $\delta_{c}^{\phi}$ and $\delta_{c}^{\chi}$ (both much bigger than $1 / R$ ) imposes fine-tuning constraints on the initial conditions, since at leading order in $1 / R$ the values for $\gamma_{\star}^{\phi}$ and $\gamma_{\star}^{\chi}$ must coincide (see eqs. (67)-(68) and recall that $p \simeq q$ ). Importantly though this kind of fine-tuning does not force us to choose a very special field value which would be inconsistent with the $\delta N$ expansion, specifically we have checked that we satisfy $\dot{\phi} \gg H^{2}$ and similarly for the $\chi$ field 33 . For more discussion of this point see [26].

We stress that the enhancement of non-Gaussianity occurs towards the end of inflation. What happens just after inflation is a model dependent issue; we cannot address this question within the approximations used in this concrete model. For our choice of parameters we notice that $f_{N L}$ is still increasing at the end of inflation, see figure 1, but for other choices it may start to decrease before inflation ends. We stress that in the regime where $\alpha, \beta \gg 1$ the slow-roll parameters will necessarily become much greater than unity by the end of inflation, which may correspond to $\left|\gamma^{s s}\right| \gg 1$ : this is one reason by which $f_{N L}$ can become large. Our

\footnotetext{
${ }^{10}$ For an example of a potentially scale-dependent local $f_{N L}$ see [26], and for a scale dependent equilateral $f_{N L}$ see for example 31. Observational prospects were recently considered in 32.
} 
formalism is at least in principle suitable to study the evolution of non-Gaussianity after inflation, when the parameter $\epsilon_{H}$ becomes larger than unity. We intend to address this issue in a future publication.

\section{Trispectrum}

In this regime where $\alpha \gg \beta>R$ and $m, n>1 / R^{2}$ it is also possible to give compact expressions for the trispectrum (4-point function) non-linearity parameters. Unlike for the bispectrum there are two nonlinearity parameters which in principle are observationally distinguishable, since they multiply terms with different shape dependence. Using the delta- $N$ formalism they are given by [15, 34, 35]

$$
\tau_{N L}=\frac{\sum_{I J K} N_{, I J} N_{, I K} N_{, J} N_{, K}}{\left(\sum_{I} N_{, I}^{2}\right)^{3}}, \quad g_{N L}=\frac{25}{54} \frac{\sum_{I J K} N_{, I J K} N_{, I} N_{, J} N_{, K}}{\left(\sum_{I} N_{, I}^{2}\right)^{3}} .
$$

We already have all the ingredients to give the full expression for $\tau_{N L}$, eqs. (29)- (33), and in principle it is a straightforward but lengthy calculation to differentiate these to get the third derivatives as required by $g_{N L}$. The full results are extremely cumbersome, but it is not hard to estimate the size of these terms, in the regime we are currently studying. Indeed it is simple to see, from eqs. (29)- (33), that at leading order in $\alpha$ the term $\partial N /\left(\partial \phi_{\star}\right)$ dominates at linear order in the delta- $N$ expansion, while at second order is the term $\partial^{2} N /\left(\partial \phi_{\star}^{2}\right)$ that dominates. With some more calculations, using the methods of Sec. 3.2, we find that the largest term at third order, at leading order in $\alpha$ and evaluated at the end of inflation, is given by

$$
\frac{\partial^{3} N}{\partial \phi_{\star}^{3}} \simeq 6 \frac{(\sqrt{2}-2 \beta \sqrt{n}) \sqrt{n}(n-m)}{\beta m^{3} n^{5}} \alpha^{5} N_{t o t}^{3} .
$$

Now also using the fact that $\beta \gg 1$ we can write the two terms for the trispectrum in terms of $f_{N L}^{2}$ as

$$
\tau_{N L} \simeq\left(\frac{6}{5} f_{N L}\right)^{2}, \quad \frac{54}{25} g_{N L} \simeq-\frac{3}{2} \frac{n-m}{m}\left(\frac{6}{5} f_{N L}\right)^{2} .
$$

The result for $\tau_{N L}$ is as expected for a model where a single field direction generates the primordial curvature perturbation [34] as it is a lower bound since in general $\tau_{N L} \geq\left(5 f_{N L} / 6\right)^{2}$ [36]. For a discussion of observational prospects for the trispectrum see [37. We note that if $m \simeq 1$ then from (65) $n \gg 1$, so the trispectrum through $g_{N L}$ will give the dominant signal of non-Gaussianity through a large, negative $g_{N L}$.

\section{Conclusions and Outlook}

We have considered models of inflation driven by multiple scalar fields with canonical kinetic terms. We studied the properties of non-Gaussianity, parameterised by the quantity $f_{N L}$ characterising the bispectrum, generated by the evolution of scalar perturbations on super Hubble scales. We developed a new method, based on a first-order Hamilton-Jacobi formalism, that provides analytical expressions for $f_{N L}$ valid in regimes beyond a slow-roll approximation. The main idea on which our arguments were based is to focus on inflationary multi-field trajectories in which the Hubble rate, and not the potential, is separable. This approach allowed us to analytically study cases where the non-Gaussian parameter $f_{N L}$ becomes large, in regimes in which the slow-roll parameters are enhanced well after Hubble exit, but before inflation ends.

By means of the same method, we also found new, exact multi-field homogeneous inflationary trajectories, for which a large amount of non-Gaussianity can be generated before the end of inflation. This allowed us to apply our general formulae on a concrete example, showing how the $f_{N L}$ parameter can grow parametrically large as a function of quantities that characterise a specific model. In the example we considered, the slowroll approximation strongly breaks down shortly before the end of inflation: $f_{\mathrm{NL}}$ grows to large values, and 
it also turns out to be scale independent. Furthermore the trispectrum is also large in this case, and for some parameter values and initial conditions the first signal of non-Gaussianity could come from a large $g_{N L}$. This is the first explicit example of a break down in slow roll after Hubble exit giving rise to $\left|f_{N L}\right| \gg 1$ at the end of inflation.

Our method and results can be applied to other contexts. Other multi-field models of inflation, besides the ones we considered, can have inflationary trajectories that satisfy our Ansatz of separable Hubble potential. All these models can be studied by means of our methods, helping to analytically understand regimes in which $f_{\mathrm{NL}}$ can be enhanced to observable values, and possibly finding new ways to enhance non-Gaussianities. In some cases, depending on the form of the potential and of the inflationary trajectory, the evolution of non-Gaussianity can be studied also after inflation ends, since our technique allows to go beyond a slow-roll approximation. For this reason it would be especially interesting to find an example of a potential with a minimum and large non-Gaussianity, so that we could address the question of how $f_{N L}$ continues to evolve after the end of the inflationary period. Also, one can straightforwardly extend our method to study multi-field inflationary models with non-canonical kinetic terms, first studied in [38]. This can allow to further explore the consequences of having non-canonical kinetic terms for the evolution of scalar fluctuations, besides what has been done so far in the literature [4,39]. We hope to return to discuss these issues soon.

Note added This version supercedes the article published in JCAP. In the previous version and the published version, there was a sign error affecting $f_{\mathrm{NL}}$ in Sec. 4.2. This was due to our previous choice of $\alpha<0$ in that section, which led to a wrong sign of $\gamma^{\phi}$ and follow on errors in several equations. We thank Ewan Tarrant and Godfrey Leung for pointing this out to us. Our results now agree with a simultaneously updated version of [40].

\section{Acknowledgments}

We thank Nelson Nunes and David Mulryne for useful discussions on related topics at the beginning of this project. Many thanks to Ki-Young Choi, Kazuya Koyama, Nelson Nunes, Filippo Vernizzi, David Wands and Ivonne Zavala for important commments on a draft of this paper. CB acknowledges financial support from the Deutsche Forschungsgemeinschaft.

\section{References}

[1] E. Komatsu et al. [WMAP Collaboration], arXiv:0803.0547 [astro-ph].

[2] E. Komatsu et al., arXiv:0902.4759 [astro-ph.CO].

[3] N. Bartolo, E. Komatsu, S. Matarrese and A. Riotto, Phys. Rept. 402, 103 (2004) arXiv:astro-ph/0406398.

[4] E. Silverstein and D. Tong, Phys. Rev. D 70, 103505 (2004) arXiv:hep-th/0310221]; M. Alishahiha, E. Silverstein and D. Tong, Phys. Rev. D 70, 123505 (2004) |arXiv:hep-th/0404084]; X. Chen, M. X. Huang, S. Kachru and G. Shiu, arXiv:hep-th/0605045; M. x. Huang and G. Shiu, Phys. Rev. D 74, 121301 (2006) arXiv:hep-th/0610235]; D. Langlois, S. Renaux-Petel, D. A. Steer and T. Tanaka, arXiv:0806.0336 [hep-th]; F. Arroja, S. Mizuno and K. Koyama, JCAP 0808, 015 (2008) arXiv:0806.0619 [astro-ph]]; X. Gao and B. Hu, arXiv:0903.1920 [astro-ph.CO]; Y. F. Cai and H. Y. Xia, Phys. Lett. B 677 (2009) 226 [arXiv:0904.0062 [hep-th]]; X. Chen, B. Hu, M. x. Huang, G. Shiu and Y. Wang, arXiv:0905.3494 [astro-ph.CO]; F. Arroja, S. Mizuno, K. Koyama and T. Tanaka, arXiv:0905.3641 [hep-th]; S. Mizuno, F. Arroja, K. Koyama and T. Tanaka, arXiv:0905.4557 [hep-th]. 
[5] N. Barnaby and J. M. Cline, JCAP 0707 (2007) 017 arXiv:0704.3426 [hep-th]]; N. Barnaby and J. M. Cline, JCAP 0806 (2008) 030 [arXiv:0802.3218 [hep-th]].

[6] J. M. Maldacena, JHEP 0305, 013 (2003) arXiv:astro-ph/0210603.

[7] D. Seery and J. E. Lidsey, JCAP 0509, 011 (2005) arXiv:astro-ph/0506056.

[8] D. Babich, P. Creminelli and M. Zaldarriaga, JCAP 0408 (2004) 009 arXiv:astro-ph/0405356; J. R. Fergusson and E. P. S. Shellard, arXiv:0812.3413 [astro-ph].

[9] F. Bernardeau and J. P. Uzan, Phys. Rev. D 66 (2002) 103506 arXiv:hep-ph/0207295]; F. Bernardeau and J. P. Uzan, Phys. Rev. D 67, 121301 (2003) arXiv:astro-ph/0209330|; F. Bernardeau and T. Brunier, Phys. Rev. D 76 (2007) 043526 [arXiv:0705.2501 [hep-ph]].

[10] D. H. Lyth, JCAP 0511, 006 (2005) [arXiv:astro-ph/0510443]; M. P. Salem, Phys. Rev. D 72, 123516 (2005) arXiv:astro-ph/0511146]; L. Alabidi and D. Lyth, JCAP 0608, 006 (2006) arXiv:astro-ph/0604569]; M. Sasaki, Prog. Theor. Phys. 120, 159 (2008) [arXiv:0805.0974 [astro-ph]]; A. Naruko and M. Sasaki, Prog. Theor. Phys. 121, 193 (2009) arXiv:0807.0180 [astro-ph]]; Q. G. Huang, JCAP 0905, 005 (2009) arXiv:0903.1542 [hep-th]]; Q. G. Huang, arXiv:0904.2649 [hep-th].

[11] L. Kofman, arXiv:astro-ph/0303614; G. Dvali, A. Gruzinov and M. Zaldarriaga, Phys. Rev. D 69, 023505 (2004) arXiv:astro-ph/0303591]; E. W. Kolb, A. Riotto and A. Vallinotto, Phys. Rev. D 71, 043513 (2005) |arXiv:astro-ph/0410546]; C. T. Byrnes and D. Wands, Phys. Rev. D 73, 063509 (2006) [arXiv:astro-ph/0512195]; K. Ichikawa, T. Suyama, T. Takahashi and M. Yamaguchi, Phys. Rev. D 78, 063545 (2008) [arXiv:0807.3988 [astro-ph]]; T. Matsuda, JCAP 0703, 003 (2007) arXiv:hep-th/0610232; T. Matsuda, JHEP 0707, 035 (2007) arXiv:0707.0543 [hep-th]]; T. Battefeld, Phys. Rev. D 77, 063503 (2008) [arXiv:0710.2540 [hep-th]]; C. T. Byrnes, JCAP 0901, 011 (2009) arXiv:0810.3913 [astro-ph]]; T. Suyama and M. Yamaguchi, Phys. Rev. D 77, 023505 (2008) arXiv:0709.2545 [astro-ph]]; K. Kohri, D. H. Lyth and C. A. Valenzuela-Toledo, arXiv:0904.0793 [hep$\mathrm{ph}$.

[12] S. Mollerach, Phys. Rev. D 42 (1990) 313; A. D. Linde and V. F. Mukhanov, Phys. Rev. D 56, 535 (1997) arXiv:astro-ph/9610219; K. Enqvist and M. S. Sloth, Nucl. Phys. B 626, 395 (2002) arXiv:hep-ph/0109214]; D. H. Lyth and D. Wands, Phys. Lett. B 524, 5 (2002) arXiv:hep-ph/0110002; T. Moroi and T. Takahashi, Phys. Lett. B 522, 215 (2001) [Erratum-ibid. B 539, 303 (2002)] arXiv:hep-ph/0110096; K. Enqvist and S. Nurmi, JCAP 0510, 013 (2005) arXiv:astro-ph/0508573; A. Linde and V. Mukhanov, JCAP 0604, 009 (2006) arXiv:astro-ph/0511736; K. A. Malik and D. H. Lyth, JCAP 0609, 008 (2006) arXiv:astro-ph/0604387]; M. Sasaki, J. Valiviita and D. Wands, Phys. Rev. D 74, 103003 (2006) arXiv:astro-ph/0607627); Q. G. Huang, JCAP 0811 (2008) 005 arXiv:0808.1793 [hep-th]]; P. Chingangbam and Q. G. Huang, JCAP 0904, 031 (2009) arXiv:0902.2619 [astro-ph.CO]].

[13] F. Vernizzi and D. Wands, JCAP 0605 (2006) 019 arXiv:astro-ph/0603799.

[14] T. Battefeld and R. Easther, JCAP 0703, 020 (2007) arXiv:astro-ph/0610296.

[15] D. Seery and J. E. Lidsey, JCAP 0701, 008 (2007) arXiv:astro-ph/0611034.

[16] K. Y. H. Choi, L. M. H. Hall and C. van de Bruck, JCAP 0702, 029 (2007) arXiv:astro-ph/0701247.

[17] C. T. Byrnes, K. Y. Choi and L. M. H. Hall, JCAP 0810 (2008) 008 [arXiv:0807.1101 [astro-ph]].

[18] G. I. Rigopoulos, E. P. S. Shellard and B. J. W. van Tent, Phys. Rev. D 76, 083512 (2007) arXiv:astro-ph/0511041. 
[19] S. Yokoyama, T. Suyama and T. Tanaka, Phys. Rev. D 77, 083511 (2008) arXiv:0705.3178 [astro-ph]]; S. Yokoyama, T. Suyama and T. Tanaka, arXiv:0711.2920 [astro-ph].

[20] D. S. Salopek and J. R. Bond, Phys. Rev. D 42 (1990) 3936.

[21] W. H. Kinney, Phys. Rev. D 56 (1997) 2002 arXiv:hep-ph/9702427].

[22] A. A. Starobinsky, JETP Lett. 42, 152 (1985) [Pisma Zh. Eksp. Teor. Fiz. 42, 124 (1985)]; M. Sasaki and E. D. Stewart, Prog. Theor. Phys. 95 (1996) 71 arXiv:astro-ph/9507001; M. Sasaki and T. Tanaka, Prog. Theor. Phys. 99, 763 (1998) arXiv:gr-qc/9801017]; D. Wands, K. A. Malik, D. H. Lyth and A. R. Liddle, Phys. Rev. D 62, 043527 (2000) arXiv:astro-ph/0003278; G. I. Rigopoulos and E. P. S. Shellard, Phys. Rev. D 68, 123518 (2003) arXiv:astro-ph/0306620]; D. H. Lyth, K. A. Malik and M. Sasaki, JCAP 0505, 004 (2005) arXiv:astro-ph/0411220].

[23] D. H. Lyth and Y. Rodriguez, Phys. Rev. Lett. 95 (2005) 121302 arXiv:astro-ph/0504045.

[24] E. Komatsu and D. N. Spergel, Phys. Rev. D 63, 063002 (2001) arXiv:astro-ph/0005036.

[25] L. Alabidi, JCAP 0610, 015 (2006) arXiv:astro-ph/0604611].

[26] C. T. Byrnes, K. Y. Choi and L. M. H. Hall, JCAP 0902, 017 (2009) arXiv:0812.0807 [astro-ph]].

[27] K. Y. Choi, J. O. Gong and D. Jeong, JCAP 0902, 032 (2009) arXiv:0810.2299 [hep-ph]].

[28] M. Sasaki, Class. Quant. Grav. 24, 2433 (2007) arXiv:astro-ph/0702182.

[29] J. P. Conlon and F. Quevedo, JHEP 0601 (2006) 146 arXiv:hep-th/0509012]; J. R. Bond, L. Kofman, S. Prokushkin and P. M. Vaudrevange, Phys. Rev. D 75 (2007) 123511 arXiv:hep-th/0612197]; A. Misra and P. Shukla, Nucl. Phys. B 800 (2008) 384 arXiv:0712.1260 [hep-th]]; M. Badziak and M. Olechowski, JCAP 0807 (2008) 021 [arXiv:0802.1014 [hep-th]]; E. Palti, G. Tasinato and J. Ward, JHEP 0806 (2008) 084 arXiv:0804.1248 [hep-th]]; H. X. Yang and H. L. Ma, JCAP 0808 (2008) 024 arXiv:0804.3653 [hep-th]].

[30] C. Gordon, D. Wands, B. A. Bassett and R. Maartens, Phys. Rev. D 63 (2001) 023506 arXiv:astro-ph/0009131.

[31] X. Chen, Phys. Rev. D 72 (2005) 123518 arXiv:astro-ph/0507053; M. LoVerde, A. Miller, S. Shandera and L. Verde, JCAP 0804, 014 (2008) [arXiv:0711.4126 [astro-ph]]; J. Khoury and F. Piazza, arXiv:0811.3633 [hep-th].

[32] E. Sefusatti, M. Liguori, A. P. S. Yadav, M. G. Jackson and E. Pajer, arXiv:0906.0232 [astro-ph.CO].

[33] P. Creminelli, S. Dubovsky, A. Nicolis, L. Senatore and M. Zaldarriaga, arXiv:0802.1067 [hep-th].

[34] C. T. Byrnes, M. Sasaki and D. Wands, Phys. Rev. D 74, 123519 (2006) arXiv:astro-ph/0611075.

[35] L. Alabidi and D. H. Lyth, JCAP 0605, 016 (2006) arXiv:astro-ph/0510441.

[36] T. Suyama and M. Yamaguchi, Phys. Rev. D 77, 023505 (2008) arXiv:0709.2545 [astro-ph]].

[37] T. Okamoto and W. Hu, Phys. Rev. D 66, 063008 (2002) arXiv:astro-ph/0206155]; N. Kogo and E. Komatsu, Phys. Rev. D 73, 083007 (2006) arXiv:astro-ph/0602099]; A. Cooray, C. Li and A. Melchiorri, Phys. Rev. D 77, 103506 (2008) arXiv:0801.3463 [astro-ph]]; D. Jeong and E. Komatsu, arXiv:0904.0497 [astro-ph.CO]. 
[38] D. A. Easson, R. Gregory, D. F. Mota, G. Tasinato and I. Zavala, JCAP 0802 (2008) 010 arXiv:0709.2666 [hep-th]].

[39] S. Renaux-Petel and G. Tasinato, JCAP 0901 (2009) 012 arXiv:0810.2405 [hep-th]].

[40] D. Battefeld, T. Battefeld, JCAP 0911, 010 (2009). arXiv:0908.4269 [hep-th]]. 